\title{
The Assessment of Serum Drug Levels to Diagnose Non-Adherence in Stable Chronic Heart Failure Patients
}

\author{
Radek Pelouch ${ }^{1,2, *}$, Viktor Voříšek ${ }^{3}$, Věra Furmanová3, Miroslav Solař ${ }^{1,2}$
}

\begin{abstract}
Background: The aim of our study was to evaluate the prevalence of drug non-adherence in stable chronic heart failure (CHF) patients using serum drug levels (SDL) assessment.

Methods: CHF patients were prospectively enrolled during scheduled outpatient visit. Except standard procedures an unanticipated blood sampling for the SDL assessment was obtained. Analysis was focused on the prescribed heart failure and antihypertensive medication and was performed by liquid chromatography coupled with mass spectrometry. The patient was labelled as non-adherent if at least one of drugs assessed was not found in the serum. In the first half of patients multiple SDL have been evaluated during the follow-up. Results: Eighty one patients were enrolled. The non-adherence was proven in twenty of them (25\%). In the subgroup of thirty eight patients with multiple SDL evaluation the non-adherence raised significantly with increasing number of visits assessed together (21\% for single visit, $29 \%$ for two of three visits assessed together and $34 \%$ for all three visits evaluated together, all $p<0.001$ ).

Conclusion: The non-adherence was proven in significant part of stable CHF patients using SDL assessment. This method seems to be reliable and effective and should be a part of clinical assessment in selected patients with CHF.

\section{KEYWORDS}

chronic heart failure; drug non-adherence; pharmacotherapy; serum drug levels

\section{AUTHOR AFFILIATIONS}

${ }^{1}$ Department of Internal Medicine, Faculty of Medicine in Hradec Králové, Charles University, Hradec Králové, Czech Republic

2 1st Department of Internal Medicine-Cardioangiology, University Hospital Hradec Králové, Czech Republic

${ }^{3}$ Institute of Clinical Biochemistry and Diagnostics, University Hospital Hradec Králové, Czech Republic

* Corresponding author: Department of Internal Medicine, Faculty of Medicine in Hradec Králové, Charles University, Šimkova 870,50038 Hradec Králové, Czech Republic; e-mail: radek.pelouch@fnhk.cz
\end{abstract}

Received: 6 January 2019

Accepted: 17 February 2019

Published online: 23 April 2019

Acta Medica (Hradec Králové) 2019; 62(2): 52-57

https://doi.org/10.14712/18059694.2019.46

(c) 2019 The Authors. This is an open-access article distributed under the terms of the Creative Commons Attribution License (http://creativecommons.org/licenses/by/4.0), which permits unrestricted use, distribution, and reproduction in any medium, provided the original author and source are credited. 


\section{INTRODUCTION}

Chronic heart failure (CHF) is a significant disease with increasing incidence and prevalence $(1,2)$. Life-saving pharmacological treatment based on Evidence based medicine remains the cornerstone of the care about CHF patients (2). Non-adherence to prescribed heart failure medication is common (3-10) and is related to higher hospitalisations and mortality rate $(3,5,6,8-10)$. To verify drug adherence may be clinically important in order to make decisions on proper treatment strategy in still symptomatic CHF patients.

However, to identify drug non-adherence may be challenging and none of the methods in use has been identified as optimal. In addition, the measurement itself is often biased by the effect of sensitizing patients and the results may be greatly affected (4). Indirect methods for drug adherence assessment include patients self-reporting (questionnaires), pill counting, and electronic medication monitoring and prescription refills (4). Monitoring systems have high specificity, but the sensitivity is reduced, because none of the methods is able to document the drug was actually ingested (11). The direct observation of drugs intake and the determination of serum or urine drug levels, using liquid chromatography coupled with mass spectrometry (LC-MS/MS) generally, are included in direct methods for drug adherence assessment (4). The reliability of direct drug measurement has been proven in studies assessing drug adherence in patients with apparent resistant hypertension (12-19). However, data in stable CHF patients are lacking.

\section{OBJECTIVE}

The aim of the study was to assess the drug non-adherence in stable CHF patients using the serum drug levels (SDL) monitoring.

\section{METHODS}

\section{SUBJECTS AND DESIGN}

We performed a prospective observational single centrum study. Consecutive CHF out-hospital patients followed on our clinic were enrolled. All participants were stable with set up CHF and antihypertensive medication. Both CHF patients with reduces and with preserved ejection fraction were suitable for the study. All patients were aged 18 years and older and gave written informed consent. The study was approved by the local Ethics committee and studies have been performed according to the Declaration of Helsinki.

The participants were enrolled to the study during scheduled outpatient visit (M1). Except standard procedures (physical examination, ECG, laboratory), an unanticipated blood sampling for the SDL measurement was performed. Analysis was focused primarily on the prescribed CHF and antihypertensive drugs, other medications were not systematically evaluated. Only drugs the patient confirmed as really intaken the evening before and the morning of the visit were considered.
According to the same rules, only in the first half of the consecutive patients multiple SDL have been evaluated during the follow-up (month 1 (at the time of enrolment, M1), month 3 (M3) and month 9 (M9).

\section{THE DETERMINATION OF SERUM DRUG LEVELS}

The determination of SDL was performed by LC-MS/MS $(20,21)$. The detection of the analysed substances was accomplished on a linear ion-trap mass spectrometer (LTQXL, Thermo Scientific, San Jose, CA, USA) using electrospray ionization. All the procedures were performed as described before $(12,22)$.

Using this precise and sensitive technique we were able to measure nearly all the spectrum of CHF and antihypertensive medications (beta-blockers, angiotensin receptor blockers, calcium channel blockers, diuretics including mineralocorticoid receptor antagonists, alpha-blockers, centrally acting drugs, digoxin and amiodarone + active metabolite). However, we were not able to assess angiotensin-converting enzyme inhibitors due to analytical limitations. Fixed drug combinations (containing maximum two drugs in our study) were considered as each component taken separately for purpose of the analysis.

INTERPRETATION OF SERUM DRUG LEVELS RESULTS Because the clinical interpretation of serum drug concentrations is difficult, any quantifiable amount of the evaluated drug was interpreted to mean that the drug was taken. Accordingly, only patient in whom the serum level of at least one drug was below the limit of detection was labelled as non-adherent. By applying this criterion for non-adherence, we eliminated uncertainties and ethical bias from the interpretation of low concentrations of drugs in the serum.

\section{STATISTICAL ANALYSIS}

Normal distributions of patients' data were evaluated with Kolmogorov-Smirnov test.

Numerical variables with a normal distribution were presented as mean \pm standard deviation. Numerical variables with a skewed distribution were presented as median (interquartile range) and categorical variables were presented as percentage (\%). Two groups comparisons of normally distributed variables were tested by unpaired t-test. The Fisher exact test was used for comparisons of non-normally distributed variables. For all tests $\mathrm{p}$-value less than 0.05 was defined as statistically significant.

\section{RESULTS}

81 consecutive patients were prospectively enrolled. The principal characteristics of the study population are summarised in Table 1. The prescribed CHF and antihypertensive medications are shown in Table 2. For each patient enrolled, 4 (median) of 8 prescribed drugs were assessed. 
Tab. 1 Principal characteristics of the study population.

\begin{tabular}{|l|l|}
\hline Number of patients & 81 \\
\hline women & $27(33 \%)$ \\
\hline Age (years) & $65(58-71)$ \\
\hline Left ventricular ejection fraction (\%) & $30(25-41)$ \\
\hline Diastolic blood presure (mmHg) & $130(120-135)$ \\
\hline Heart rate (beats per min) & $75(70-81)$ \\
\hline Weight (kg) (mean \pm SD) & $71(65-82)$ \\
\hline Body mass index & $93 \pm 17$ \\
\hline NT-proBNP (pg/ml) & $31(27-34)$ \\
\hline Diabetes mellitus & $702(304-2212)$ \\
\hline Arteral hypertension & $33(41 \%)$ \\
\hline Atrial fibrilation & $63(78 \%)$ \\
\hline Etiology of CHF & $11(14 \%)$ \\
\hline dilatated cardiomyopathy & \\
\hline ischemic heart disease & $31(39 \%)$ \\
\hline other cardiomyopathy & $21(26 \%)$ \\
\hline significant valvular disease & $6(7 \%)$ \\
\hline multifactorial & $4(5 \%)$ \\
\hline unknown & $14(17 \%)$ \\
\hline
\end{tabular}

Unless stated otherwise, the data are expressed as median (25-75\%). Individual diagnosis are expressed as number of patients (\% of study population).

Tab. 2 Prescribed CHF and antihypertensive medications.

\begin{tabular}{|l|l|}
\hline BetaBlockers & $71(88 \%)$ \\
\hline $\begin{array}{l}\text { Angiotensin-converting enzyme } \\
\text { inhibitors }\end{array}$ & $48(59 \%)$ \\
\hline Angiotensin receptor blockers & $14(17 \%)$ \\
\hline Furosemide & $52(64 \%)$ \\
\hline Thiazides & $52(64 \%)$ \\
\hline Aldosterone antagonists & $57(70 \%)$ \\
\hline Calcium channel blockers & $18(23 \%)$ \\
\hline AlphaBlockers & $8(10 \%)$ \\
\hline Centrally acting drugs & $2(3 \%)$ \\
\hline Ivabradine & $3(4 \%)$ \\
\hline Amiodarone & $23(28 \%)$ \\
\hline Digoxin & $2(3 \%)$ \\
\hline Warfarin & $30(37 \%)$ \\
\hline Statins & $50(62 \%)$ \\
\hline
\end{tabular}

Number of patients with prescribed individual drug/drug class (\% of study population).
All of evaluated drugs were detected in the serum of 61 patients (75\%) and the criteria for non-adherence were fulfilled in the remaining 20 patients (25\%). One of all drugs was undetectable in 10 patients (12.5\%), more than one in the serum of 9 patients (11\%). None of the evaluated drugs was detectable in 1 patient (1.5\%). The results of the adherence assessment are summarised in Table 3. The adherence and non-adherence according to individual drugs/drug classes are shown in Table 4 and Figure 1.

Tab. 3 Adherence assessment.

\begin{tabular}{|l|l|}
\hline Number of patients & 81 \\
\hline Number of prescribed drugs & $8(6-10)$ \\
\hline Number of drugs evaluated & $4(3-4)$ \\
\hline Number of adherent patients & $61(75 \%)$ \\
\hline Number of non-adheretn patients & $20(25 \%)$ \\
\hline one of all drugs undetectable & $10(12.5 \%)$ \\
\hline more than one of all drugs undetectable & $9(11 \%)$ \\
\hline none of all drugs indetectable & $1(1.5 \%)$ \\
\hline
\end{tabular}

Number of prescribed and evaluated drugs is expressed as median (25-75\%).

Number of patients (\% of study population).

Tab. 4 Non-adherence according to individual drugs/drug classes.

\begin{tabular}{|l|l|}
\hline BetaBlockers & $9 \%(6 / 71)$ \\
\hline Angiotensin receptor blockers & $14 \%(2 / 14)$ \\
\hline Furosemide & $10 \%(5 / 48)$ \\
\hline Thiazides & $12 \%(6 / 52)$ \\
\hline Aldosterone antagonists & $9 \%(5 / 57)$ \\
\hline Calcium channel blockers & $28 \%(5 / 18)$ \\
\hline AlphaBlockers & $13 \%(1 / 8)$ \\
\hline Centrally acting drugs & $0 \%(0 / 2)$ \\
\hline Amiodarone & $9 \%(2 / 23)$ \\
\hline Digoxin & $0 \%(0 / 2)$ \\
\hline
\end{tabular}

Non-adherence is expressed as percentage of patients with undetectable drug of total number of evaluated patients for individual drug/drug class the respective numbers (undetectable/total number) are in the brackets. Furosemide was evaluated only if it was confirmed by patient as intaken in the morning.

Multiple SDL were planned to be assessed in the first 40 consecutive patients. However, two patients died during the course of the study therefore only data of 38 patients were analysed finally (Table 5 ). If each single visit $(\mathrm{M} 1 / 3 / 9)$ assessed separately the non-adherence was proven in $21 \%$ of patients in this subgroup and was similar to the non- adherence of the whole study population $(25 \%$, $\mathrm{p}=0.24$ ). The non-adherence assessed for two of three visits together $(M 1+2, M 1+3, M 2+3$, adherence defined as all drugs detectable in both blood-samplings) was proven in $29 \%$ and finally the non-adherence assessed for all three visits together $(\mathrm{M} 1+3+9$, adherence defined as all drugs detectable in all blood-samplings) was proven in $34 \%$ of 
patients. With an increasing number of visits evaluated together the frequency of non-adherence has raised significantly (all $\mathrm{p}<0.001$ ).

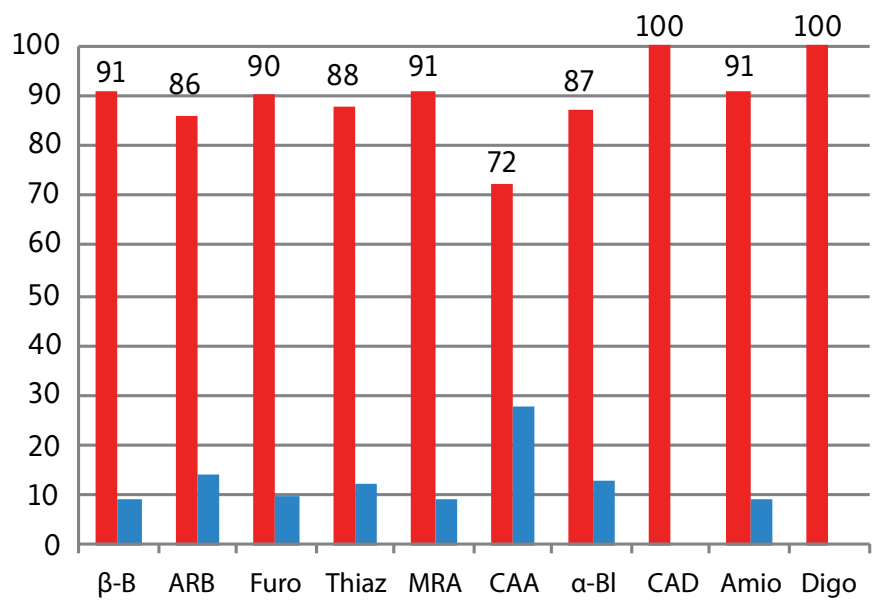

$\square$ Adherence (\%) $\quad$ Non-adherence (\%)

Fig. 1 Adherence/Non-adherence according to individual drugs/ drug classes.

Abbreviations: $\beta$-B-BetaBlockers; ARB-Angiotensin receptor blockers; Furo - Furosemide; Thiaz - Thiazides; MRA - Aldosterone antagonists; CAA - Calcium channel blockers; a-Bl - AlphaBlockers; CAD - Centrally acting drugs; Amio - Amiodarone; Digo - Digoxin.

\section{DISCUSSION}

Non-adherence to prescribed medication has been reported in $11-60 \%$ CHF patients (3-10) as well as its negative effect on acute decompensations and mortality rate $(3,5,6,8-10)$. Finally, the negative economic impact of non-adherence to CHF medication has been also proven $(23,24)$.

However, to recognise drug non-adherence may be challenging in daily clinical practice. In CHF patients the adherence has been evaluated on the basis of pharmacy and insurance records analysis (prescription refills) or on patients self-reporting (questionnaires) in the previous studies $(3-10,23,24)$. Unfortunately, the sensitivity of self-reporting is reduced (4), which has been documented

Tab. 5 Non-adherence in subgroup with multiple SDL evaluations.

\begin{tabular}{|c|c|}
\hline Number of controls evaluated together & Non-adherence \\
\hline Single control (median) & $8 / 38(21 \%)$ \\
\hline$M 1$ & $8 / 38(21 \%)$ \\
\hline$M 3$ & $7 / 38(18 \%)$ \\
\hline$M 9$ & $8 / 38(21 \%)$ \\
\hline Two controls (median) & $11 / 38(29 \%)$ \\
\hline$M 1+3$ & $11 / 38(29 \%)$ \\
\hline$M 1+9$ & $12 / 38(32 \%)$ \\
\hline$M 3+9$ & $10 / 38(26 \%)$ \\
\hline Three controls $(M 1+3+9)$ & $13 / 38(34 \%)$ \\
\hline
\end{tabular}

Numbers of non-adherent patients/total number of patient in subgroup (\% in the brackets). in retrospective study with apparent drug resistant arterial hypertension patients. Only nearly half of non-adherent patients was identified using one of the most frequently used questionnaire The Morisky Medication Adherence Scale (MMAS-8) compared to SDL evaluated by LC-MS/ MS (19). On the other hand, prescription refills is a method much more suitable for research purpose than for clinical evaluation $(4,25)$. The suitability and the reliability of direct drug monitoring have been demonstrated in several studies with patients who presented with difficult-to-control arterial hypertension. Using drug monitoring in the serum $(12-15,18,19)$ or in the urine $(16,17)$ the non-adherence has been reported in $25-86 \%$ of these patients.

Moreover, the British and Czech authors have shown that screening for non-adherence using LC-MS/MS and subsequent results discussion with the non-adherent hypertensive patients led to improved drug adherence and significant blood pressure drop (26). In addition, a recent study has demonstrated this approach as cost-saving strategy in patients with apparent resistant hypertension (27).

Based on the above listed data, drug monitoring can be considered as suitable method for drug adherence assessing in patients with apparent resistant arterial hypertension. However, to the best of our knowledge, the use of this method in stable CHF patients has not been reported before. We have only proved the non-adherence in significant part (44\%) of patients presenting with acute decompensated heart failure using SDL monitoring in our previous study (22).

In the present study we have identified the non-adherence in 20 of $81(25 \%)$ CHF patients. Compared to previous studies $(5,6,8,9)$, the frequency of non-adherence was relatively low and the adherence to individual drug classes including diuretics and beta-blockers was relatively high in our study. We can only speculate about reasons. It could be explained by the fact that only stable patients in long-term follow-up with gradually titrated medication were enrolled to the study. By this way some of known unfavourable influences, such as drug related site effects or doctor-patient relationship related factors could be eliminated.

It is obvious that a single SDL assessment provides information about actual adherence only and it does not have to correspond to long-term cooperation. This idea is in line with our results. In subgroup of the first 38 consecutive patients multiple SDL evaluations have been performed (month 1, 3 and 9) and the frequency of non-adherence has risen significantly with an increasing number of visits evaluated together. Assessing single visit, the non-adherence was proven in $21 \%$ of patients, for two visits together in $29 \%$ and for all three visits in $34 \%$ of patients (all $\mathrm{p}<0.001$ ). Unfortunately, even multiple SDL monitoring cannot rule out "white-coat adherence" thought those patients, who took their medication only before scheduled visits.

The qualitative assessment of SDL could be another reason for possible drug non-adherence omission in our study (4). Any detectable amount of drug was interpreted as a proof of adherence, therefore it was not able to identify patients taking the medication irregularly or in 
lower doses (4). However, the quantitative evaluation of drug levels is difficult in daily clinical practice. Serum and urine concentrations may vary widely between-individual and within-individual due to fluctuations in drugs pharmacokinetics, genetic polymorphisms (cytochromes $\mathrm{P} 450$, drug transporters etc.), disease-induced poor absorption, and renal elimination or drug-drug interactions (4). In addition, it is generally impossible to ensure suitable time interval between drug intake and unanticipated blood sampling during the out-hospital visits. The combination of drug monitoring and pharmacokinetic simulations seem to be a possible solution of this problem $(4,18)$, unfortunately probably too complicated for daily clinical practise.

Another limitation of our study was the exclusion of angiotensin converting enzyme inhibitors from the analysis. Evaluation of active metabolites is necessary for reliable assessment of this drug class, which was not available in our hospital at the time the study was performed.

The extension of drug monitoring for adherence assessment may be limited by its cost and technical requirements. But, the drug non-adherence identification may have important clinical consequences in still symptomatic patients with set up medication who are generally candidates for other expensive therapeutic options (resynchronization, mechanical circulatory support, heart transplantation). Non-adherence evidence should lead to close physician-patients cooperation with maximal effort to improve the adherence instead of extension of other therapy first of all. As well as in apparent resistant hypertonic patients (27), we can suppose this approach to be cost-effective in CHF patients. But this assumption has to be confirmed by further studies.

\section{CONCLUSION}

Based on serum drug levels monitoring, non-adherence was proven in significant part of stable chronic heart failure patients. We believe this method is reliable and effective in drug adherence evaluation and should be a part of clinical assessment in still symptomatic patients with chronic heart failure, particularly before searching for new therapeutic options.

\section{ACKNOWLEDGEMENTS}

This work was supported by the research project PRVOUK 037/03.

\section{CONFLICT OF INTEREST}

The authors declare no conflict of interest.

\section{REFERENCES}

1. Van Riet EES, Hoes1 AW, Wagenaar KP, Limburg A, Landman MAJ and Rutten FH. Epidemiology of heart failure: the prevalence of heart failure and ventricular dysfunction in older adults over time. A systematic review. European Journal of Heart Failure 2016; 18: 242-52.
2. Ponikowski P, Voors AA, Anker SD, et al. 2016 ESC Guidelines for the diagnosis and treatment of acute and chronic heart failure. European Heart Journal 2016; 37: 2129-200.

3. Riles EM, Jain AV, Fendrick AM. Medication Adherence and Heart failure. Curr Cardiol Rep 2014; 16: 458.

4. Hamdidouche I, Jullien V, Boutouyrie P, Billaud E, Azizi M, Laurent S. Drug adherence in hypertension: from methodological issues to cardiovascular outcomes. Journal of Hypertension 2017; 35: 1133-44.

5. Gislason GH, Rasmussen JN, Abildstrom SZ, et al. Persistent Use of Evidence-Based Pharmacotherapy in Heart Failure Is Associated With Improved Outcomes. Circulation 2007; 116: 737-44.

6. Fitzgeralg AA, Powers JD, Ho PM, et al. Impact of Medication Nonadherence on Hospitalizations and Mortality in Heart Failure. J Cardiac Fail 2011; 17: 664-9.

7. Zhang Y, Wu SH, Fendrick AM, Baicker K. Variation in Medication Adherence in Heart Failure. JAMA Intern Med 2013; 173(6): 468-70.

8. Yoo BS, Oh J, Hong BK, et al. SUrvey of Guideline Adherence for Treatment of Systolic Heart Failure in Real World (SUGAR): A Multi-Center, Retrospective, Observational Study. PLoS ONE 2014; 9: 1-7.

9. Hood SR, Giazzon AJ, Seamon G, et al. Association Between Medication Adherence and the Outcomes of Heart Failure. Pharmacotherapy 2018; 38(5): 539-45.

10. Granger BB, Swedberg K, Ekman I, et al. Adherence to candesartan and placebo and outcomes in chronic heart failure in the CHARM programme: doubleblind, randomised, controlled clinical trial. Lancet 2005; 366: 2005-11.

11. Van Onzenoort HA, Verberk WJ, Kessels AG, et al. Assessing Medication Adherence Simultaneously By Electronic Monitoring And Pill Count In Patients With Mild-To-Moderate Hypertension. Am J Hypertens 2010; 23(2): 149-54.

12. Ceral J, Habrdova V, Vorisek V, Bima M, Pelouch R, Solar M. Difficult-to-control arterial hypertension or uncooperative patients? The assessment of serum antihypertensive drug levels to differentiate non-responsiveness from non-adherence to recommended therapy. Hypertension Research 2011; 34: 87-90.

13. Florczak E, Tokarczyk B, Warchoł-Celińska E, et al. Assessment of adherence to treatment in patients with resistant hypertension using toxicological serum analysis. A subgroup evaluation of the RESISTPOL study. Pol Arch Med Wewn 2015; 125(1-2): 65-72.

14. Avataneo V, De Nicolò A, Rabbia F, et al. Therapeutic drug monitoringguided definition of adherence profiles in resistant hypertension and identification of predictors of poor adherence. Br J Clin Pharmacol 2018; 84: 2535-43.

15. Strauch B, Petrak O, Zelinka T, et al. Precise assessment of noncompliance with the antihypertensive therapy in patients with resistant hypertension using toxicological serum analysis. Journal of Hypertension 2013; 31: 2455-61.

16. Jung O, Gechter JL, Wunder C, et al. Resistant hypertension? Assessment of adherence by toxicological urine analysis. Journal of Hypertension 2013; 31: 766-74.

17. Tomaszewski $M$, White $C h$, Patel $P$, et al. High rates of non-adherence to antihypertensive treatment revealed by high-performance liquid chromatography-tandem mass spektrometry (HP LC-MS/MS) urine analysis. Heart 2014; 100(11): 855-61.

18. Šíma M, Vodička M, Marešová V, Šálek T, Čabala R, Slanař O. Adherence with perindopril therapy: a pilot study using therapeutic drug monitoring of perindoprilat and an evaluation of the clearance estimation. Int J Clin Pharm 2017; 39: 1095-100.

19. Pandey A, Raza F, Velasco A, et al. Comparison of Morisky Medication Adherence Scale with therapeutic drug monitoring in apparent treatment-resistant hypertension. Journal of the American Society of Hypertension 2015; 9: 420-6.

20. Maurer HH. Multi-analyte procedures for screening for and quantification of drugs in blood, plasma, or serum by liquid chromatography-single stage or tandem mass spectrometry (LC-MS or LC-MS/ MS) relevant to clinical and forensic toxicology. Clin Biochem 2005; 38: 310-8.

21. Kolocouri F, Dotsikas Y, Apostolou C, Kousoulos C, Loukas YL. Simultaneous determination of losartan, EXP-3174 and hydrochlorothiazide in plasma via fully automated 96 -well-format-based solid-phase extraction and liquid chromatography-negative electrospray tandem mass spectrometry. Anal Bioanal Chem 2007; 387: 593-601.

22. Solar M, Pelouch R, Vorisek V, Furmanova V, Ceral J. Serum drug levels to diagnose non-adherence in acute decompensated heart failure. Biomed Pap Med Fac Univ Palacky Olomouc Czech Repub 2016; 160(2): 244-7.

23. Roebuck MCh, Liberman JN, Gemmill-Toyama M, Brennan TA. Medication adherence leads to lower health care use and costs despite increased drug spending. Health Affairs 2011; 30(1): 91-9. 
24. Muszbek N, Brixner D, Benedict A, Keskinaslan A, Khan ZM. The economic consequences of noncompliance in cardiovascular disease and related conditions: a literature review. Int J Clin Pract 2008; 62(2): 338-51.

25. Zullig LL, Mendys P, Bosworth HB. Medication adherence: A practical measurement selection guide using case studies. Patient Education and Counseling 2017; 100: 1410-4.
26. Gupta P, Patel P, Štrauch B, et al. Biochemical Screening for Nonadherence Is Associated With Blood Pressure Reduction and Improvement in Adherence. Hypertension 2017; 70: 1042-8.

27. Schoonhoven AV, Asselt ADI, Tomaszewski M, et al. Cost-Utility of an Objective Biochemical Measure to Improve Adherence to Antihypertensive Treatment. Hypertension 2018; 72: 1117-24. 